\title{
Fatal lymphocytic myocarditis masquerading as thoracic aortic dissection
}

\author{
Eugene Sharma Henry \\ Emergency Department, The Mid Yorkshire Hospitals NHS Trust, Wakefield, UK
}

\section{Keywords}

Emergency medicine $\cdot$ Resuscitation $\cdot$ Cardiology $\cdot$ Decision-making

\begin{abstract}
Acute myocarditis usually presents with a broad spectrum of symptoms and has variable clinical outcomes. A proportion of acute myocarditides may develop into fulminant myocarditis resulting in significant mortality if left untreated. This case report describes an unusual presentation of fatal lymphocytic myocarditis mimicking a thoracic aortic dissection in a previously healthy 45-year-old male. Fulminant myocarditis should be considered as part of the differential diagnosis in an acutely unwell young adult presenting to the Emergency Department with non-specific symptoms.
\end{abstract}

\section{Introduction}

Viral myocarditis has an incidence rate of $0.01-0.02 \%$, may present with a self-limiting clinical picture or conversely, and may have fatal outcomes [1]. Despite the low incidence rate, it is responsible for $10-20 \%$ of sudden cardiac deaths amongst young adults [2]. Lymphocytic myocarditis (LM) rarely causes sudden cardiac death as compared to giant cell myocarditis. Review articles [3,4] and individual case reports [5-7] conclude that the presenting complaints for patients with biopsy-confirmed myocarditis are non-specific and are difficult to diagnose clinically. The presentation may mimic an infection from an alternative focus such as a urinary tract infection or an acute myocardial infarction. 
Henry: Fatal lymphocytic myocarditis masquerading as thoracic aortic dissection

\section{Case Presentation}

A 45-year-old male initially presented to the Emergency Department (ED) complaining of abdominal pain, diarrhoea, and a sudden "pop" feeling in the stomach. Blood tests were done at triage, and the patient was placed in the queue to be seen by a clinician.

The patient left the department with the aim of returning when it was less crowded. Investigation results and observations in this case report are presented in Table 1.

A junior doctor noted that the patient's blood results were grossly deranged and immediately recalled the patient. The patient reattended the department and complained that his hands and feet were feeling numb that and his tongue was swelling up. He attributed the tongue swelling to the Ibuprofen tablet that he took. The patient looked very unwell, and he was immediately triaged to the resuscitation room for further management.

The patient denied any recent infective illnesses. There was no significant past medical history. The positive findings on physical examination were swollen tongue and weak peripheral upper limb pulses. Cardiovascular and respiratory examination was unremarkable. Abdomen was soft non-tender. His ECG showed tall and tented T waves (Fig. 1). Chest X-ray was normal.

The patient was treated initially as an anaphylactic reaction, and the hyperkalaemia was corrected. Blood pressure reading of both upper limbs showed a difference of $12 \mathrm{~mm}$ Hg systolic (right arm 139/95 and left arm 127/98). A bedside point of care echocardiography showed poor cardiac contractility (eyeballing 15-25\%) with no right heart strain. These findings were confirmed by a cardiologist. The intensive care team was involved at this point as the patient continued to deteriorate (looking more mottled), and he was transferred to the radiology department for a computed tomography (CT) angiogram of the thorax and abdomen with full monitoring. The CT was verbally reported as no large pulmonary embolus, and there were features suggestive of a dissecting thoracic aorta (Fig. 2). Both radial arteries were non-palpable. Patient's blood pressure dropped to $60 / 40 \mathrm{~mm} \mathrm{Hg}$ and intravenous inotrope boluses were given without any satisfactory response. As the blood gas was unsurvivable and the working diagnosis was thoracic aortic dissection, the resuscitation team made the decision not to perform cardiopulmonary resuscitation on the patient. The patient died in the department, $2 \mathrm{~h}$ after his reattendance to the ED. The case was referred to the coroner's office to investigate the cause of death. COVID-19 PCR swab was negative.

\section{Differential Diagnosis}

This case proved to be a diagnostic challenge as the patient presented with a number of symptoms which did not particularly fit into a disease-specific symptomology. A systematic approach was used to address each symptom to further tease out the possible diagnosis. Life-threatening pathologies were treated immediately. The main differential diagnoses included (i) pulmonary embolism or (ii) thoracic aortic dissection or (iii) fulminant sepsis. In the context of hypotension, the absence of right heart strain on bedside echocardiography and a negative $\mathrm{CT}$, the diagnosis of a massive pulmonary embolism was excluded. With the feeble peripheral pulses on the upper limb, blood pressure difference, and high D - Dimer result, the diagnosis of thoracic aortic dissection was felt to be the most likely option. This suspicion was further supported with the hyperlactaemia which was believed to be caused by the hypo-perfused state in critical aortic dissection. The formal CT report was made available just after the patient died (Table 1). The cause of death was deemed by the coroner to be acute LM of viral aetiology. Myocarditis was not considered as a differential diagnosis from the outset.

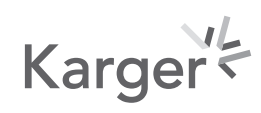


Table 1. Investigations and observations table

Investigations
Observations (at triage)
Observations ( in resuscitation room )

Arterial blood gas (done on arrival at resuscitation room)

Arterial blood gas (preterminal)

Haematology

Biochemistry

D - dimer

CT

Post-mortem report

\section{Results}

Heart rate: 86

Blood pressure: 114/80 mm Hg

Saturations: $99 \%$

Temperature: $37^{\circ} \mathbf{C}$

Heart rate: 89

Blood pressure: 136/112 mm Hg

Saturations: unrecordable

Temperature: $35.4^{\circ} \mathrm{C}$

PH: 7.30, pCO2: 2.3 kPa, p02 15.4 kPa, Sodium 125 mmol/L, Potassium $7.3 \mathrm{mmol} / \mathrm{L}$, bicarbonate $8.4 \mathrm{mmol} / \mathrm{L}$, base excess - $15 \mathrm{mmol} / \mathrm{L}$, lactate $8.3 \mathrm{mmol} / \mathrm{L}$

pH < 6.80, pCO2 7.2 kPa, p02 $7.2 \mathbf{~ k P a , ~ s o d i u m ~} 127 \mathbf{m m o l} / \mathbf{L}$, potassium $7.6 \mathrm{mmol} / \mathrm{L}$, bicarbonate $=$ incalculable, base excess $=$ incalculable, lactate $13.6 \mathrm{mmol} / \mathrm{L}$

Haemoglobin: $160 \mathrm{~g} / \mathrm{L}$ range (130-180)

White Cell Count: $6.910^{9} / \mathrm{L}$ range $(4.3-13.0)$

Platelet count: $20 \mathbf{1 0}^{9} / \mathrm{L}$ range (135-400)

APTT: $58.1 \mathrm{~s}$ range (22-32)

PT: $17.3 \mathrm{~s}$ range (9.5-12.5)

Fibrinogen: 1.7 g/L (1.5-4.5)

Troponin: haemolysed sample

Urea: $6.5 \mathrm{mmol} / \mathrm{L}$ range (2.2-7.7)

Creatinine: $134 \mathbf{~ m m o l} / \mathrm{L}$ range (59-104)

Sodium: $134 \mathbf{~ m m o l} / \mathrm{L}$ range (136-145)

Potassium: haemolysed

Alanine aminotransferase: $101 \mu / \mathrm{L}$ range (0-56)

Bilirubin: $56 \mu \mathrm{mol} / \mathrm{L}$ range (0-21)

Blood film results

Severe thrombocytopenia. Some polychromatic cells. Spherocytes present. Some nucleated red cells present. Howell Jolly Bodies present. Neutrophils appear left shifted. Vacuolated neutrophils present.

$59.1 \mathrm{mg} / \mathrm{L}$ (normal range < $0.5 \mathrm{mg} / \mathrm{L}$ )

Concerns about an aortic dissection, however, the changes could also be due to mixing of unopacified stagnant contrast to the significant hypotension. The changes within the lungs were reported as atypical for heart failure and could be related to poor cardiac output conditions such as myocarditis Normal aorta with mild left ventricular hypertrophy and biventricular dilatation. Tissue samples from the heart showed several small foci of interstitial lymphocytic inflammation associated with myocyte loss. No other infection foci

CT, computed tomography.

\section{Discussion}

This is a rare case of a patient presenting with fulminant LM masquerading initially, as a thoracic aortic dissection. This patient presented with normal observations and then very rapidly deteriorated. The derangement of the blood tests suggested multi-organ failure 


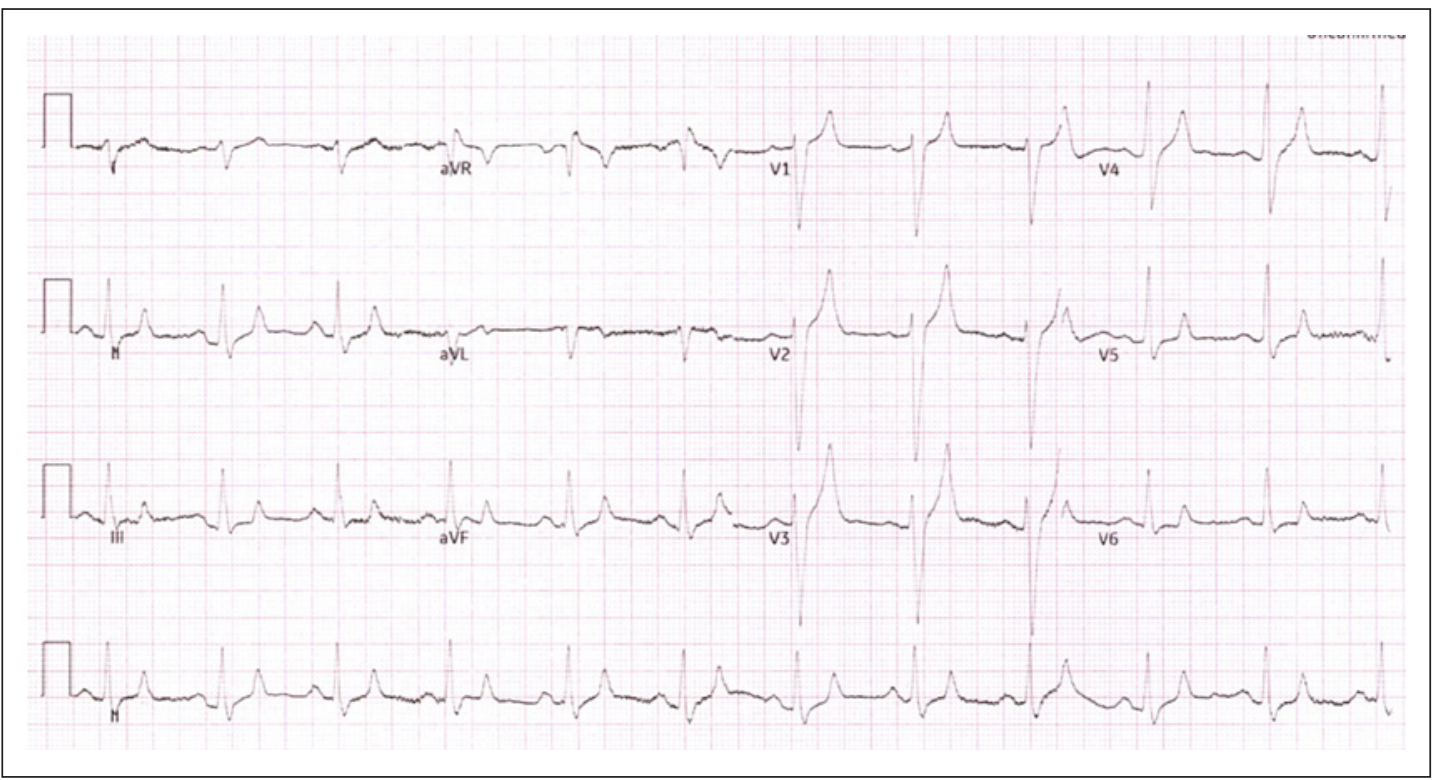

Fig. 1. ECG showing tall and tented T waves.

Fig. 2. Key image from the CT scan showing a possible dissecting thoracic aorta. The marking here denotes a linear low-density region within the aortic arch. CT, computer tomography.

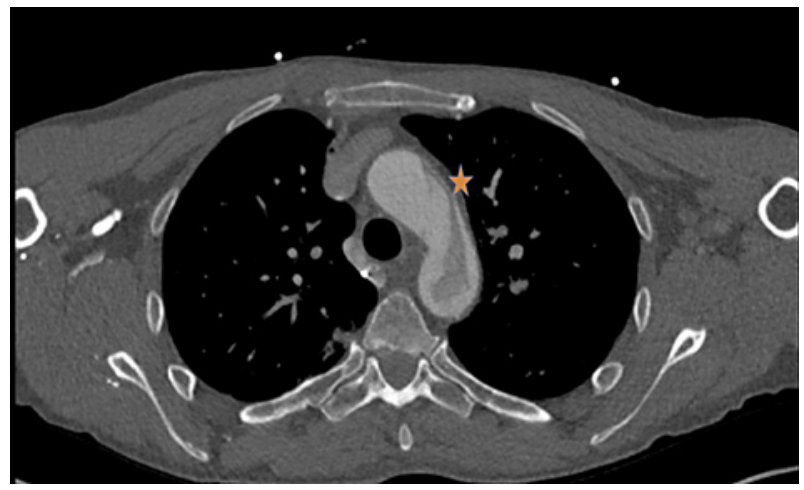

(deranged liver function tests, blood gas, and electrolytes). It is noted that fulminant sepsis could be a possible cause of death here with myocarditis being a bystander. The post-mortem report did not identify any septic foci.

Fulminant myocarditis is associated with the mortality rate of $>50 \%$ [8]. The treatment strategy for fulminant myocarditis includes aggressive hemodynamic support (extracorporeal membrane oxygenation or ventricular assist device) [9]. The role of immunosuppressive therapy and antiviral therapy in the acute phase of fulminant myocarditis has yet to prove a clear benefit [10]. Case series [11] suggest that hemodynamically unstable patients with fulminant myocarditis have paradoxically favourable outcomes when treated with aggressive hemodynamic support in specialized centres [12]. However, the in-hospital mortality rates remain high [8]. Due to the rarity of the disease, larger datasets are lacking $[12,13]$. Prompt diagnosis remains key as patients with fulminant LM have a favourable outcome with the right treatment compared to other histological forms of myocarditis [14].

Given the complexity of the clinical presentation, a high index of suspicion (or prior exposure) is required to be able to consider the rare diagnosis of fulminant myocarditis as part of the differential diagnosis. Through the lens of the dual-process decision-making 
theory, the type of judgement involved is attributed to System 1 (or intuitive) thinking [15]. Therefore, prior exposure and pattern recognition are crucial in order to derive the diagnosis in time to offer the appropriate treatment.

In conclusion, fulminant myocarditis should be considered as a possible differential diagnosis in an acutely unwell young adult presenting with non-specific symptoms. Early recognition could possibly direct life-saving treatment strategies despite significant hemodynamic embarrassment. Nevertheless, it is prudent to mention that it is still a diagnostic dilemma to differentiate the disease with other similar emergencies such as sepsis, in the context of uncertainty in the ED.

\section{Learning Points}

- The degree of diagnostic uncertainty in decision-making for Emergency Medicine is high. This warrants a low threshold to recall patients when there are clinical concerns.

- Acute myocarditis is an established cause of sudden cardiac death amongst young adults. The best way of suspecting it is through pattern recognition from prior exposure or education and/or training.

- Point of care ultrasound remains an important rule-in tool for life-threatening pathologies in the undifferentiated patient.

\section{Statement of Ethics}

Written consent has been given by the next of kin for the publication of this case. Images have been reviewed by the next of kin prior to publication. This manuscript is exempt from Ethical Committee approval as it is not defined as a research by the NHS Health Research Authority.

\section{Conflict of Interest Statement}

The author has no conflicts of interest to declare.

\section{Funding Sources}

The author did not receive any funding.

\section{Author Contributions}

EH conducted the literature review and wrote the manuscript.

\section{Availability of Data and Material}

All available data has been anonymized and written consent has been obtained for publication. The histopathological slides have been disposed prior to the write-up at the explicit request of the next of kin. 


\section{References}

1 Olejniczak M, Schwartz M, Webber E, Shaffer A, Perry TE. Viral myocarditis-incidence, diagnosis and management. J Cardiothorac Vasc Anesth. 2020;34(6):1591-601.

2 Cooper LT Jr. Ventricular arrhythmias and sudden cardiac death in lymphocytic myocarditis. Washington DC: American College of Cardiology Foundation; 2020.

3 Shauer A, Gotsman I, Keren A, Zwas DR, Hellman Y, Durst R, et al. Acute viral myocarditis: current concepts in diagnosis and treatment. Isr Med Assoc J. 2013;15(3):180-5.

4 Kytö V, Saukko P, Lignitz E, Schwesinger G, Henn V, Saraste A, et al. Diagnosis and presentation of fatal myocarditis. Hum Pathol. 2005;36(9):1003-7.

5 Giafaglione JR, Morrison AK, Nandi D. A case report of myocarditis masquerading as hypertrophic cardiomyopathy. Ann Pediatr Cardiol. 2020 Oct-Dec;13(4):340.

6 Chrysohoou C, Tsiamis E, Brili S, Barbetseas J, Stefanadis C. Acute myocarditis from coxsackie infection, mimicking subendocardial ischaemia. Hellenic J Cardiol. 2009;50(2):147-50.

7 Spartalis M, Tzatzaki E, Spartalis E, Damaskos C, Mavrogeni S, Voudris V. Parvovirus B19 myocarditis of fulminant evolution. Cardiol Res. 2017;8(4):172-5.

8 Ammirati E, Cipriani M, Lilliu M, Sormani P, Varrenti M, Raineri C, et al. Survival and left ventricular function changes in fulminant versus nonfulminant acute myocarditis. Circulation. 2017;136(6):529-45.

9 McCarthy RE, Boehmer JP, Hruban RH, Hutchins GM, Kasper EK, Hare JM, et al. Long-term outcome of fulminant myocarditis as compared with acute (Nonfulminant) myocarditis. N Engl J Med. 2000;342(10):690-5.

10 Cuomo V, Esposito R, Santoro C. Fulminant myocarditis in the time of coronavirus. Eur Heart J. 2020;41(22): 2121.

11 Lee CH, Tsai WC, Hsu CH, Liu PY, Lin LJ, Chen JH. Predictive factors of a fulminant course in acute myocarditis. Int J Cardiol. 2006;109(1):142-5.

12 Hang W, Chen C, Seubert JM, Wang DW. Fulminant myocarditis: a comprehensive review from etiology to treatments and outcomes. Signal Transduct Target Ther. 2020;5(1):287.

13 Ammirati E, Cipriani M, Camici PG. New concepts in fulminant myocarditis and risk of cardiac mortality. Oncotarget. 2017;8(49):84624-5.

14 Kociol RD, Cooper LT, Fang JC, Moslehi JJ, Pang PS, Sabe MA, et al. Recognition and initial management of fulminant myocarditis: a scientific statement from the American Heart Association. Circulation. 2020;141(6): e69-92.

15 Croskerry P. A universal model of diagnostic reasoning. Acad Med. 2009;84(8):1022-8. 